\title{
Bose Condensed Gas in Strong Disorder Potential With Arbitrary Correlation Length
}

\author{
Patrick Navez ${ }^{1,2}$, Axel Pelster ${ }^{1}$, Robert Graham ${ }^{1}$ \\ ${ }^{1}$ Universitaet Duisburg-Essen, Universitaet Duisburg-Essen, Lotharstrasse 1, 47048 Duisburg, Germany, \\ ${ }^{2}$ Labo Vaste-Stoffysica en Magnetisme, Katholieke Universiteit Leuven, \\ Celestijnenlaan 200 D, B-3001 Heverlee, Belgium
}

(Dated: July 8, 2018)

\begin{abstract}
We study the properties of a dilute Bose condensed gas at zero temperature in the presence of a strong random potential with arbitrary correlation length. Starting from the underlying GrossPitaevskii equation, we use the random phase approximation in order to get a closed integral equation for the averaged density distribution which allows the determination of the condensate and the superfluid density. The obtained results generalize those of Huang and Meng (HM) to strong disorder. In particular, we find the critical value of the disorder strength, where the superfluid phase disappears by a first-order phase transition. We show how this critical value changes as a function of the correlation length.
\end{abstract}

PACS numbers: 03.75.Hh,03.75.Kk,05.30.-d

\section{INTRODUCTION}

An ultracold atom gas in the presence of disordered environments is becoming a subject of increasing experimental and theoretical research activities. Generally, one would like to understand how the condensation and the superfluid properties of ultracold gases are influenced by a spatially random force on the atoms. In some experiments, the random potential is created by optical means to show its effects on the transport properties of a Bose gas [1, 2, 3]. In most others, however, one must rather face the reality of unavoidable external random forces which are induced either by the roughness of a dielectric surface [4], by the magnetic field along wires with current irregularities [5], or by different localized atomic species [6]. Furthermore, recent theoretical results on the impact of randomness on bosons in lattices are reviewed in Refs. [7, 8].

Most theoretical studies on a 3D disordered Bose gas are limited to calculations up to the second-order in the random potential. In these works, the weak disorder induces only small corrections to the condensate depletion, the superfluid density [9], the collective excitations and their damping [10], and the condensation critical point [11]. The extension to strong disorder has so far been analysed only numerically in Ref. 12]. More recently, an analytical mean-field study [13], which takes into account higher order corrections, has shown the possibility of having a transition from a superfluid phase to a Bose glass phase where the spatial long-range correlations have completely disappeared.

In this paper, we address the issue of the influence of strong disorder at zero temperature for a finite correlation length [14, 15]. The random potential follows a Gaussian distribution and is said to be uncorrelated in the case where all Fourier components contribute equally to the randomness, while it is correlated when the influence of the Fourier components falls off for wavenumbers larger the inverse correlation length $\xi$. As encoutered in experiments [5], we choose a Lorentzian correlation function. Usually, this length appears to be much bigger than the healing length and thus affects the condensate properties.

In order to simplify this physical problem, we assume that all particles occupy the same quantum state, for which the macroscopic wave-function obeys the GrossPitaevskii equation in the presence of an external spatially random force. In order to solve this stochastic nonlinear differential equations, we apply the random phase approximation (RPA) 16] and take the ensemble average over all possible realisations of the associated potential. In the clean case without a random potential, this gapless and conserving approximation has been successfully used in the context of calculating of the collective excitations at finite temperatures 17,18 and in kinetic theory [19]. In our case with disorder, we obtain the particle density distribution beyond the lowest order expansion in the random potential. With this we extend the seminal work of Ref. [9] to strong disorder, which has the consequence that the superfluid phase disappears by a first-order transition. We show how the critical value of the disorder strength, for which this transition occurs, depends on the correlation length.

\section{MOMENTUM DISTRIBUTION}

We start from the Gross-Pitaevskii equation at zero temperature written in Fourier space. Defining the Fourier components according to $\psi(\mathbf{r})=$ $\sum_{\mathbf{k}} e^{i \mathbf{k} . \mathbf{r}} \alpha_{\mathbf{k}} / \sqrt{V}$, we get in units with $\hbar=1$ :

$$
\begin{aligned}
\left(i \frac{\partial}{\partial t}-\frac{\mathbf{k}^{2}}{2 m}\right) \alpha_{\mathbf{k}}= & \sum_{\mathbf{q}} U_{\mathbf{q}} \alpha_{\mathbf{k}-\mathbf{q}} \\
& +\frac{g}{V} \sum_{\mathbf{k}^{\prime}, \mathbf{q}} \alpha_{\mathbf{k}^{\prime}}^{*} \alpha_{\mathbf{k}+\mathbf{q}} \alpha_{\mathbf{k}^{\prime}-\mathbf{q}},
\end{aligned}
$$

where the random potential $U_{\mathbf{q}}$ follows a Gaussian distribution $\left\langle U_{\mathbf{q}} U_{\mathbf{q}^{\prime}}\right\rangle=\delta_{\mathbf{q},-\mathbf{q}^{\prime}} R(\mathbf{q}) / V$. The quadratic amplitude is assumed to be Lorentzian $R(\mathbf{q})=R /\left(1+\xi^{2} \mathbf{q}^{2}\right)$, 
i.e. it is correlated below the wavenumber $1 / \xi$. We assume that a macroscopic fraction of the condensate moves with a velocity $\mathbf{k}_{\mathbf{s}} / m$.

In order to derive a dynamic dielectric function for the total fluid, we define the bilinear combination

$$
\rho_{\mathbf{k}, \mathbf{q}}=\alpha_{\mathbf{k}}^{*} \alpha_{\mathbf{k}+\mathbf{q}} .
$$

It represents an excitation of momentum $\mathbf{q}$ created from a particle which releases its momentum from $\mathbf{k}+\mathbf{q}$ to k. In particular, it allows the definition of the Fourier component of the density fluctuation: $\rho_{\mathbf{q}}=\sum_{\mathbf{k}} \rho_{\mathbf{k}, \mathbf{q}}=$ $\rho_{-\mathbf{q}}^{*}$. The dynamic evolution of (2) following from (11) is given by:

$$
\begin{aligned}
& i \frac{\partial}{\partial t} \rho_{\mathbf{k}, \mathbf{q}}=\left(\epsilon_{\mathbf{k}+\mathbf{q}}-\epsilon_{\mathbf{k}}\right) \rho_{\mathbf{k}, \mathbf{q}}+ \\
& \sum_{\mathbf{q}^{\prime}}\left(U_{\mathbf{q}^{\prime}}+\frac{g}{V} \sum_{\mathbf{k}^{\prime}} \alpha_{\mathbf{k}^{\prime}}^{*} \alpha_{\mathbf{k}^{\prime}+\mathbf{q}^{\prime}}\right)\left(\alpha_{\mathbf{k}}^{*} \alpha_{\mathbf{k}+\mathbf{q}-\mathbf{q}^{\prime}}-\alpha_{\mathbf{k}+\mathbf{q}^{\prime}}^{*} \alpha_{\mathbf{k}+\mathbf{q}}\right) .
\end{aligned}
$$

The technical details for implementing the random phase approximation in the clean case can be found either in Ref. [16] or in Ref. [19] where a finite-temperature Bose gas has been considered with $\rho_{\mathbf{k}, \mathbf{q}}$ being a quantum operator. Here, we shall briefly repeat this procedure by considering random variables instead of operators.

We treat the quartic terms in (3) within a factorisation procedure and use the property that $\left\langle\rho_{\mathbf{k}, \mathbf{q}}\right\rangle=0$ for $\mathbf{q} \neq 0$ in an homogeneous gas. For any quartic term, we approximate

$$
\begin{aligned}
& \alpha_{\mathbf{k}_{1}}^{*} \alpha_{\mathbf{k}_{2}}^{*} \alpha_{\mathbf{k}_{3}} \alpha_{\mathbf{k}_{4}}-\left\langle\alpha_{\mathbf{k}_{1}}^{*} \alpha_{\mathbf{k}_{2}}^{*} \alpha_{\mathbf{k}_{3}} \alpha_{\mathbf{k}_{4}}\right\rangle \simeq \\
& \quad+\left\langle\left|\alpha_{\mathbf{k}_{1}}\right|^{2}\right\rangle \delta_{\mathbf{k}_{1}, \mathbf{k}_{3}} \alpha_{\mathbf{k}_{2}}^{*} \alpha_{\mathbf{k}_{4}} \\
& \quad+\left\langle\left|\alpha_{\mathbf{k}_{2}}\right|^{2}\right\rangle \delta_{\mathbf{k}_{2}, \mathbf{k}_{4}}\left(1+\delta_{\mathbf{k}_{1}, \mathbf{k}_{2}} \delta_{\mathbf{k}_{3}, \mathbf{k}_{4}}\right) \alpha_{\mathbf{k}_{1}}^{*} \alpha_{\mathbf{k}_{3}} \\
& \quad+\left\langle\left|\alpha_{\mathbf{k}_{1}}\right|^{2}\right\rangle \delta_{\mathbf{k}_{1}, \mathbf{k}_{4}}\left(1-\delta_{\mathbf{k}_{1}, \mathbf{k}_{2}}-\delta_{\mathbf{k}_{3}, \mathbf{k}_{4}}\right) \alpha_{\mathbf{k}_{2}}^{*} \alpha_{\mathbf{k}_{3}} \\
& \quad+\left\langle\left|\alpha_{\mathbf{k}_{2}}\right|^{2}\right\rangle \delta_{\mathbf{k}_{2}, \mathbf{k}_{3}}\left(1-\delta_{\mathbf{k}_{1}, \mathbf{k}_{2}}-\delta_{\mathbf{k}_{3}, \mathbf{k}_{4}}\right) \alpha_{\mathbf{k}_{1}}^{*} \alpha_{\mathbf{k}_{4}}
\end{aligned}
$$

which avoids double counting. For example, in case of $\mathbf{k}_{\mathbf{1}}=\mathbf{k}_{\mathbf{2}}$ and $\mathbf{k}_{\mathbf{3}} \neq \mathbf{k}_{\mathbf{4}}$ the approximation reduces to two terms only which is important for contributions involving the macroscopic component $\mathbf{k}_{\mathbf{s}}$ of the condensate. Since the average over the quartic term in Eq.(4) applied in (3) involves components with the total transfer momentum $\mathbf{q} \neq 0$, it will not contribute for an homogeneous gas.

Through this procedure, the RPA keeps among all terms those combinations involving products of offdiagonal terms $\rho_{\mathbf{k}^{\prime}, \mathbf{q}}$ and averaged diagonal ones $n_{\mathbf{k}^{\prime \prime}}=$ $\left\langle\left|\alpha_{\mathbf{k}^{\prime \prime}}\right|^{2}\right\rangle$ for all possible values of $\mathbf{k}^{\prime}$ and $\mathbf{k}^{\prime \prime}$, and neglects all others combinations. Therefore, we remove contributions which are bilinear in $\rho_{\mathbf{k}^{\prime}, \mathbf{q}^{\prime}}$ for $\mathbf{q}^{\prime} \neq \mathbf{q}, \mathbf{0}$. In this way, we obtain the linear integral equation for $\mathbf{q} \neq \mathbf{0}$ :

$$
\begin{array}{r}
{\left[i \frac{\partial}{\partial t}-\left(\epsilon_{\mathbf{k}+\mathbf{q}}-\epsilon_{\mathbf{k}}\right)\right] \rho_{\mathbf{k}, \mathbf{q}}=} \\
\left(U_{\mathbf{q}}+\frac{g \rho_{\mathbf{q}}}{V}\right)\left(n_{\mathbf{k}}-n_{\mathbf{k}+\mathbf{q}}\right)+\frac{g \rho_{\mathbf{q}}}{V}\left(n_{\mathbf{k}}^{\prime}-n_{\mathbf{k}+\mathbf{q}}^{\prime}\right) \\
+\left(1-\delta_{\mathbf{k}, \mathbf{k}_{\mathbf{s}}}-\delta_{\mathbf{k}, \mathbf{k}_{\mathbf{s}}-\mathbf{q}}\right) \sum_{\mathbf{k}^{\prime} \neq \mathbf{k}_{\mathbf{s}}, \mathbf{k}_{\mathbf{s}}-\mathbf{q}} \frac{g \rho_{\mathbf{k}^{\prime}, \mathbf{q}}}{V} n_{\mathbf{k}_{\mathbf{s}}} .
\end{array}
$$

Here $n_{\mathbf{k}}^{\prime}=\left(1-\delta_{\mathbf{k}_{\mathbf{s}}, \mathbf{k}}\right) n_{\mathbf{k}}$ refers to the disordered part of the condensate which consists of all its part which are not at the wavenumber $\mathbf{k}_{\mathbf{s}}$. Note at this stage that $\rho_{\mathbf{k}, \mathbf{q}}$ is still a random variable. Thus, we should still take the ensemble average over any non-vanishing combination like $\left\langle U_{-\mathbf{q}} \rho_{\mathbf{k}, \mathbf{q}}\right\rangle$ and $\left\langle\rho_{\mathbf{k}^{\prime},-\mathbf{q}} \rho_{\mathbf{k}, \mathbf{q}}\right\rangle$ and solve the resulting equations. Equivalently, here we directly solve Eq. (5) for $\rho_{\mathbf{k}, \mathbf{q}}$ and perform the disorder average at a later stage.

In order to make the link with the dielectric formalism, let us assume for the moment that the potential has a temporal dependence of the form $U_{\mathbf{q}}(t)=$ $\exp (-i \omega t) U_{\mathbf{q}, \omega}$. Then the solution is of the form $\rho_{\mathbf{k}, \mathbf{q}}(t)=\exp (-i \omega t) \rho_{\mathbf{k}, \mathbf{q}, \omega}$ as well. Under these conditions, we find a solution:

$$
\begin{aligned}
& \rho_{\mathbf{k}, \mathbf{q}, \omega}=\frac{\left(U_{\mathbf{q}, \omega}+2 g \rho_{\mathbf{q}, \omega} / V\right)\left(n_{\mathbf{k}}-n_{\mathbf{k}+\mathbf{q}}\right)}{\omega+i 0_{+}-\left(\epsilon_{\mathbf{k}+\mathbf{q}}-\epsilon_{\mathbf{k}}\right)} \\
& \times \frac{\left(\omega+i 0_{+}-\frac{\mathbf{k}_{\mathbf{s}} \mathbf{q}}{m}\right)^{2}-\left(\frac{\mathbf{q}^{2}}{2 m}\right)^{2}}{\left(\omega+i 0_{+}-\frac{\mathbf{k}_{\mathbf{s}} \mathbf{q}}{m}\right)^{2}-\left(\frac{\mathbf{q}^{2}}{2 m}\right)^{2}+\frac{g n_{\mathbf{k}} \mathbf{q}^{2}}{m V}\left(\delta_{\mathbf{k}, \mathbf{k}_{\mathbf{s}}}+\delta_{\mathbf{k}, \mathbf{k}_{\mathbf{s}}-\mathbf{q}}\right)}
\end{aligned}
$$

which is similar to Ref. [19]. The Fourier component of the density fluctuations can therefore be written in the form:

$$
\rho_{\mathbf{q}, \omega}=\chi(\mathbf{q}, \omega) U_{\mathbf{q}, \omega},
$$

with the susceptibility

$$
\chi(\mathbf{q}, \omega)=\frac{V}{2 g}\left[\frac{1}{\mathcal{K}(\mathbf{q}, \omega)}-1\right] .
$$

The dynamic dielectric function $\mathcal{K}(\mathbf{q}, \omega)$ defined by (8) for the total fluid can be decomposed into [19]

$$
\begin{aligned}
\mathcal{K}(\mathbf{q}, \omega) & =\mathcal{K}_{n}(\mathbf{q}, \omega) \\
& +\frac{-\frac{2 g n_{\mathbf{k}_{\mathbf{s}}}}{V} \frac{\mathbf{q}^{2}}{m}}{\left(\omega+i 0_{+}-\frac{\mathbf{k}_{\mathbf{s}} \cdot \mathbf{q}}{m}\right)^{2}-\left(\frac{\mathbf{q}^{2}}{2 m}\right)^{2}+\frac{g n_{\mathbf{k}_{\mathbf{s}}} \mathbf{q}^{2}}{m V}},
\end{aligned}
$$

where we obtain for the disordered part of the fluid:

$$
\mathcal{K}_{n}(\mathbf{q}, \omega)=1-\frac{2 g}{V} \sum_{\mathbf{k}} \frac{n_{\mathbf{k}}^{\prime}-n_{\mathbf{k}+\mathbf{q}}^{\prime}}{\omega+i 0_{+}-\frac{\mathbf{k} \cdot \mathbf{q}}{m}-\frac{\mathbf{q}^{2}}{2 m}},
$$

(cf. also 19, 20]). In this way, we recover the same expression as in Ref. [17, 20] for the susceptibility function $\chi(\mathbf{q}, \omega)$.

In the special case of a time-independent potential $U_{\mathbf{q}, \omega}=U_{\mathbf{q}} \delta_{\omega, 0}$ which is of interest here, the solution (6) can be used to define a self-consistent relation that allows the calculation of $n_{\mathbf{k}}^{\prime}$. At first, combining Eqs. (6)-(10), we get the response function for a particle of the condensate to be excited with momentum q:

$$
\rho_{\mathbf{k}_{\mathbf{s}}, \mathbf{q}}=n_{\mathbf{k}_{\mathbf{s}}} \frac{U_{\mathbf{q}}}{\left(i 0_{+}-\frac{\mathbf{k}_{\mathbf{s}} \cdot \mathbf{q}}{m}-\frac{\mathbf{q}^{2}}{2 m}\right) \tilde{\mathcal{K}}(\mathbf{q})} .
$$


For $\omega=0$, the screening factor $\tilde{\mathcal{K}}(\mathbf{q})$ defined by (11) for any external force acting on the condensate particles is:

$$
\begin{aligned}
& \tilde{\mathcal{K}}(\mathbf{q})= \\
& \frac{-\left(\frac{\mathbf{k}_{\mathbf{s}} \cdot \mathbf{q}}{m}\right)^{2}+\left(\frac{\mathbf{q}^{2}}{2 m}\right)^{2}}{\mathcal{K}_{n}(\mathbf{q}, 0)\left[\epsilon_{\mathbf{q}}^{B^{2}}-\left(\frac{\mathbf{k}_{\mathbf{s}} \cdot \mathbf{q}}{m}\right)^{2}\right]-\left[\mathcal{K}_{n}(\mathbf{q}, 0)-1\right] \frac{2 g n_{\mathbf{k}_{\mathbf{s}}} \mathbf{q}^{2}}{m V}} .
\end{aligned}
$$

In the simple special case $n_{\mathbf{k}}^{\prime}=0$, i.e. $\mathcal{K}_{n}(\mathbf{q}, 0)=1$ and $\mathbf{k}_{\mathbf{s}}=0$, we obtain more simply:

$$
\tilde{\mathcal{K}}(\mathbf{q})=\frac{1}{1+\left(4 m g n_{0} / V\right) / \mathbf{q}^{2}} .
$$

This formula shows that the random force acting on the condensate particles is screened for momenta below the inverse of the healing length which plays here the role of the Debye length for the condensate.

Let us now notice that: $\left\langle\rho_{\mathbf{k}_{\mathbf{s}}, \mathbf{q}}^{*} \rho_{\mathbf{k}_{\mathbf{s}}, \mathbf{q}}\right\rangle=$ $\left\langle\left|\alpha_{\mathbf{k}_{\mathbf{s}}}\right|^{2}\left|\alpha_{\mathbf{k}_{\mathbf{s}}+\mathbf{q}}\right|^{2}\right\rangle=n_{\mathbf{k}_{\mathbf{s}}} n_{\mathbf{k}_{\mathbf{s}}+\mathbf{q}}^{\prime}$ for $\mathbf{q} \neq 0$ whereas the fluctuations of the macroscopic $\mathbf{k}_{\mathbf{s}}$ component around the average are small in the thermodynamic limit. Using this relation in (11) and (12), we arrive at the following non-linear integral equation for $n_{\mathbf{k}_{\mathbf{s}}+\mathbf{q}}^{\prime}$ :

$$
\begin{aligned}
& n_{\mathbf{k}_{\mathbf{s}}+\mathbf{q}}^{\prime}= \\
& \frac{R(\mathbf{q})\left(\epsilon_{\mathbf{k}_{\mathbf{s}}-\mathbf{q}}-\epsilon_{\mathbf{k}_{\mathbf{s}}}\right)^{2} n_{\mathbf{k}_{\mathbf{s}}} / V}{\left|\mathcal{K}_{n}(\mathbf{q}, 0)\left[\epsilon_{\mathbf{q}}^{B}-\left(\frac{\mathbf{k}_{\mathbf{s}} \cdot \mathbf{q}}{m}\right)^{2}\right]-\left(\mathcal{K}_{n}(\mathbf{q}, 0)-1\right) \frac{2 g n_{\mathbf{k}_{\mathbf{s}}}}{V} \frac{\mathbf{q}^{2}}{m}\right|^{2}},
\end{aligned}
$$

where $\left.\epsilon_{\mathbf{q}}^{B}=\sqrt{c_{B}^{2} \mathbf{q}^{2}+\left(\mathbf{q}^{2} / 2 m\right.}\right)^{2}$ denotes the Bogoliubov excitation energy and $c_{B}=\sqrt{g n_{\mathbf{k}_{\mathbf{s}}} / m V}$ is the sound velocity in the absence of disorder. The nonlinearity comes from the fact that the dieletric function $\mathcal{K}_{n}(\mathbf{q}, 0)$ depends via Eq. (10) on $n_{\mathbf{k}_{\mathbf{s}}+\mathbf{q}}^{\prime}$. Let us first consider the case of weak disorder where we can approximate $\mathcal{K}_{n}(\mathbf{q}, 0) \simeq 1$ and recover the second-order result for the disordered components of the condensate $[9]$ :

$$
n_{\mathbf{k}_{\mathbf{s}}+\mathbf{q}}^{\prime}=\frac{R(\mathbf{q})\left(\epsilon_{\mathbf{k}_{\mathbf{s}}-\mathbf{q}}-\epsilon_{\mathbf{k}_{\mathbf{s}}}\right)^{2} n_{\mathbf{k}_{\mathbf{s}}} / V}{\left[\epsilon_{\mathbf{q}}^{B^{2}}-\left(\frac{\mathbf{k}_{\mathbf{s}} \cdot \mathbf{q}}{m}\right)^{2}\right]^{2}} .
$$

This formula is regular provided the Landau stability criterion $\epsilon_{\mathbf{q}}^{B}>\left|\mathbf{k}_{\mathbf{s}} \cdot \mathbf{q} / m\right|$ is satisfied. An increase of $\mathbf{k}_{\mathbf{s}}$ would increase the disordered part of the fluid until a singularity is reached leading to an instability. For $\mathbf{k}_{\mathbf{s}}=0$, we recover the weak-disorder result for this part [9]:

$$
\sum_{\mathbf{q}} n_{\mathbf{q}}^{\prime}=\sum_{\mathbf{q}} \frac{R(\mathbf{q}) n_{\mathbf{0}} / V}{\left[\epsilon_{\mathbf{q}}^{2}+2 g n_{\mathbf{0}} / V\right]^{2}}=R \frac{m^{3 / 2}}{4 \pi^{2}} \sqrt{\frac{V}{g n_{0}}} n_{0} .
$$

The superfluid fraction $n_{s}$ is defined as the part of the fluid which is moving at a velocity $\mathbf{k}_{\mathbf{s}} / m$ and is found by calculating the total momentum of the gas:

$$
\mathbf{P}=\sum_{\mathbf{k}} \mathbf{k} n_{\mathbf{k}}=\mathbf{k}_{\mathbf{s}} n_{s}
$$

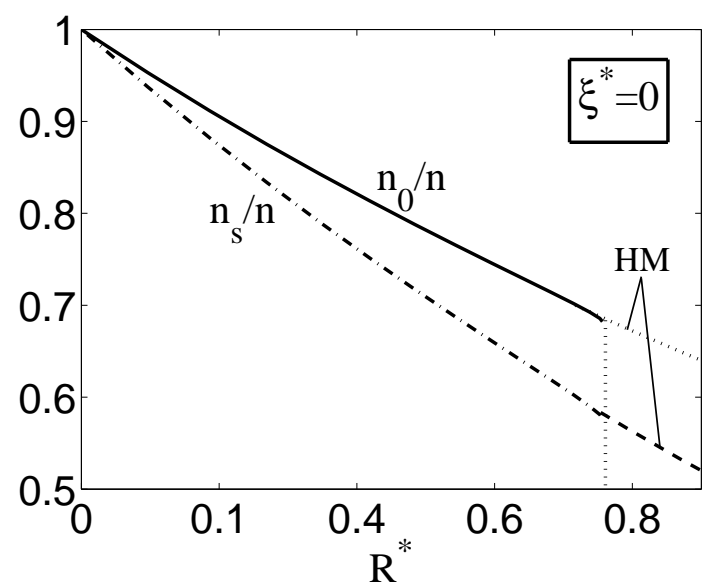

FIG. 1: Clean part of the condensate fraction $n_{\mathbf{0}}$ as a function of $R^{*}$ in the case of uncorrelated disorder in the RPA model (full curve) and in the HM model (dotted curve) and the corresponding superfluid fraction $n_{s}$ (dot-dashed curve in RPA and dashed curve in HM model).

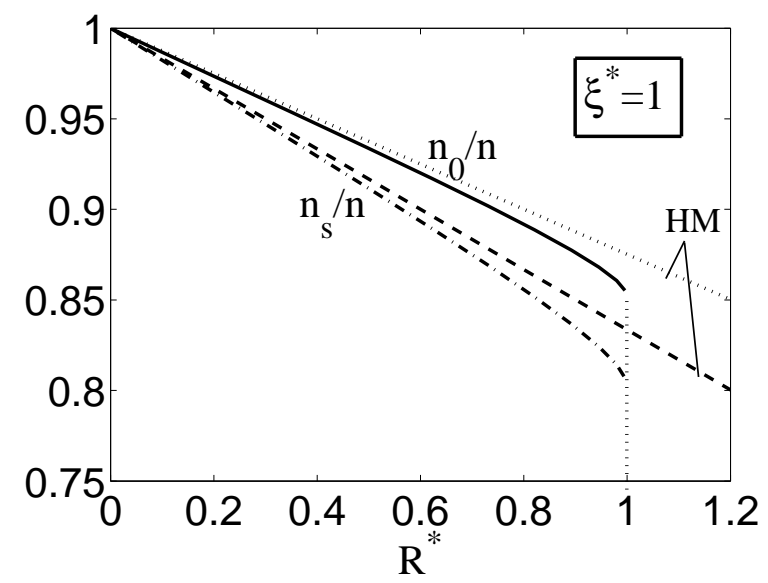

FIG. 2: Same as for Fig.1 but for correlated disorder with $\xi^{*}=1$.

In the limit of small velocity $\mathbf{k}_{\mathbf{s}} / m$, we get the expression:

$$
n-n_{s}=\left.\frac{1}{3} \sum_{\mathbf{k}}\left(\mathbf{k} \cdot \frac{\partial}{\partial \mathbf{k}_{\mathbf{s}}} n_{\mathbf{k}_{\mathbf{s}}+\mathbf{k}}\right)\right|_{\mathbf{k}_{\mathbf{s}}=0} .
$$

Noticing that $\mathcal{K}_{n}(\mathbf{q}, 0)$ with the solution $n_{\mathbf{k}}^{\prime}$ is an even function of $\mathbf{k}_{\mathbf{s}}$, we deduce from Eq. (14):

$$
n-n_{s}=\frac{4}{3}\left(n-n_{0}\right)
$$

This relation is identical to the one in Ref. [9] but is here found to remain valid in the RPA for the more general case of strong disorder. 


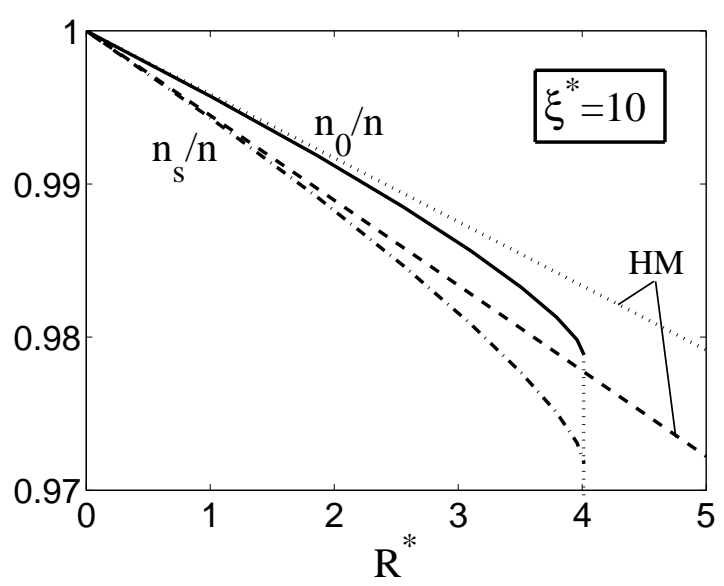

FIG. 3: Same as for Fig.1 but for correlated disorder with $\xi^{*}=10$.

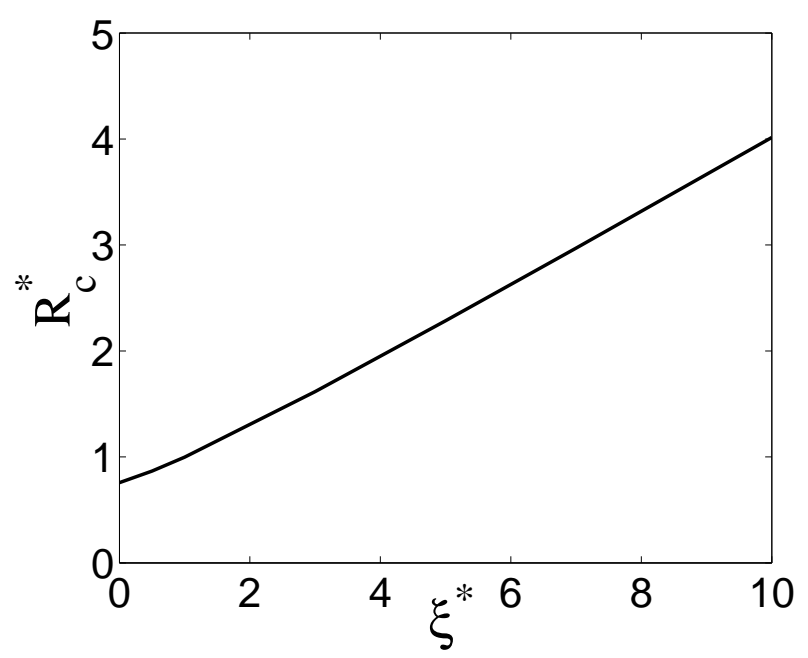

FIG. 4: Critical value for the disorder intensity $R_{c}^{*}$ as a function of the correlation length $\xi^{*}$.

\section{PHASE TRANSITION}

Equation (14) is solved numerically by an iterative procedure as a function of the reduced dimensionless parameters for the disorder strength $R^{*}=R m^{3 / 2} / \sqrt{4 \pi^{2} g n / V}$ and the correlation length $\xi^{*}=\sqrt{4 m g n / V} \xi$. We have looked for the stable solution that minimizes the total energy of the system. The homogeneous part of the condensate $n_{0}$ and the superfluid fraction $n_{s}$ are plotted in Figs. 1-3 as a function of the reduced disorder strength $R^{*}$ and are compared with the results obtained by HM. The transition is determined as the highest value $R^{*}=R_{c}^{*}$ for which the condensate fraction $n_{0}$ is nonzero so that spatial coherence is preserved over the entire space. This point corresponds to the situation where $n_{0}$ has an infinite derivative with respect to $R^{*}$. As a consequence of relation (19), also the superfluid density $n_{s}$ has there an infinite derivative. For uncorrelated disorder i.e. $\xi^{*}=0$, we notice only a tiny difference with respect to the second-order theory, until we reach the critical value when the disordered part of the condensate is about 30 percent. For higher values of $\xi^{*}$ this difference becomes more pronounced, but a smaller fraction of the disordered part of the fluid is needed in order to achieve the transition. Fig. 4 shows that the critical value $R_{c}^{*}$ increases as a function of the coherence length which is understandable from the fact that for larger $\xi^{*}$ the high spatial frequency components of the disordered part of the condensate are smaller (cf. Eq.(14)).

\section{CONCLUSIONS}

The random phase approximation has been applied to the Gross-Pitaevskii equation in the presence of a random potential in order to describe a strongly disordered Bose gas at zero temperature. This approximation goes beyond a previous second-order calculation and predicts a first-order phase transition from a superfluid phase to a non-superfluid phase. The critical value of the disorder intensity for this transition depends strongly on the correlation length. Nevertheless, our model fails to describe the properties of the non-superfluid phase. A possible explanation is that the assumption of a unique wave function for any particle excludes the possibility of having fragmented condensates for strong disorder that could be necessary in a such a phase.

\section{Acknowledgements}

This work was supported by the SFB/TR 12 of the German Research Foundation (DFG). Furthermore, PN acknowledges support from the german $\mathrm{AvH}$ foundation, from the Belgian FWO project 3E050202, and from Junior Fellowship F/05/011 of the KUL Research Council.
[1] D. Clément, A. F. Varon , M. Hugbart, J. Retter, P. Bouyer, L. Sanchez-Palencia, D. M. Gangardt, V. Shlyapnikov, and A. Aspect, Phys. Rev. Lett. 95, 170409 (2005).

[2] L. Fallani, J. E. Lye, V. Guarrera, C. Fort, and M. Inguscio, eprint: cond-mat/0603655 C. Fort, L. Fallani, V.
Guarrera, J. E. Lye, M. Modugno, D. S. Wiersma, and M. Inguscio Phys. Rev. Lett. 95, 170410 (2005); J. E. Lye, L. Fallani, M. Modugno, D. S. Wiersma, C. Fort, and M. Inguscio Phys. Rev. Lett. 95, 070401 (2005) .

[3] T. Schulte, S. Drenkelforth, J. Kruse, W. Ertmer, J. Arlt, K. Sacha, J. Zakrzewski, and M. Lewenstein Phys. Rev. 
Lett. 95, 170411 (2005).

[4] H. Perrin, Y. Colombe, B. Mercier, V. Lorent, and C. Henkel, J. Phys. B 19, 151 (2005).

[5] R. Folman, P. Krüger, J. Schmiedmayer, J. Denschlag, and C. Henkel, Adv. At. Mol. Opt. Phys. 48, 263 (2002).

[6] S. Ospelkaus, C. Ospelkaus, O. Wille, M. Succo, P. Ernst, K. Sengstock, and K. Bongs, Phys. Rev. Lett. 96, 180403 (2006).

[7] K.V. Krutitsky, A. Pelster, and R. Graham, New J. Phys. 8, 187 (2006).

[8] M. Lewenstein, A. Sanpera, V. Ahufinger, B. Damski, A.S. De, and U. Sen, eprint: cond-mat/0606771.

[9] K. Huang and Meng, Phys. Rev. Lett. 69, 644 (1992).

[10] S. Giorgini, L. Pitaevskii, and S. Stringari, Phys. Rev. B 49, 12938 (1994).

[11] A. V. Lopatin and V.M. Vinokur, Phys. Rev. Lett. 88, 235503 (2002).

[12] G.E. Astrakharchik, J. Boronat, J. Casulleras, and S.
Giorgini, Phys. Rev. A 66, 023603 (2002).

[13] R. Graham, and A. Pelster, eprint: cond-mat/0508306

[14] M. Kobayashi and M. Tsubota, Phys. Rev. B 66, 174516 (2002).

[15] M. Timmer, A. Pelster, and R. Graham, Europhys. Lett. 76, 760 (2006).

[16] D. Pines and P. Nozières, Theory of Quantum Liquids, Vol. 1 (Benjamin, 1966).

[17] M. Fliesser, J. Reidl, P. Szépfalusy, and R. Graham, Phys. Rev. A 64, 013609 (2001).

[18] J. Reidl, A. Csordas, R. Graham, and P. Szépfalusy, Phys. Rev. A 61, 043606 (2000).

[19] P. Navez, Physica A 356, 241 (2005); P. Navez, J. Low Temp. Phys. 138, 705 (2005).

[20] P. Navez and R. Graham, Phys. Rev. A 73, 043612 (2006). 